\begin{tabular}{|c|c|}
\hline Title & EELS A nalysis of Oxygen Scavenging Effect in a Resi stive Switching Structure of Pt/Ta/SrTi03Pt \\
\hline Author(s) & T surumaki-Fukuchi, A tsushi; Nakagawa, Ryosuke; A rita, Masashi; T akahashi, Y asuo \\
\hline Citation & $\begin{array}{l}\text { MRS A dvances, 3(33), 1925-1930 } \\
\text { https://doi.org/10.1557/adv.2018.12 }\end{array}$ \\
\hline Issue Date & 2018 \\
\hline Doc URL & http:/hdl.handle.net/2115/72301 \\
\hline Rights & $\begin{array}{l}\text { This article has been published in a revised form in MRS A dvances, https://doi .org/10.1557/adv.2018.12. This version } \\
\text { is free to view and download for private research and study only. Not for re-distribution, re-sale or use in derivative } \\
\text { works. (C copyright holder. }\end{array}$ \\
\hline Tyре & article (author version) \\
\hline File Information & A tsushi Fukuchi, MRS A dvances.pdf \\
\hline
\end{tabular}

Instructions for use 


\title{
EELS Analysis of Oxygen Scavenging Effect in a Resistive Switching Structure of $\mathrm{Pt} / \mathrm{Ta} / \mathrm{SrTiO}_{3} / \mathrm{Pt}$
}

\author{
Atsushi Tsurumaki-Fukuchi, Ryosuke Nakagawa, Masashi Arita and Yasuo Takahashi
}

Graduate School of Information Science and Technology, Hokkaido University, Sapporo, Hokkaido 0600814, Japan

ABSTRACT

A complex mechanism of interfacial oxygen scavenging is revealed by electron energy-loss spectroscopy (EELS) for a resistive switching oxide of $\mathrm{SrTiO}_{3}$ with a scavenging layer of Ta. When Ta thin layer is inserted at one of the interfaces of $\mathrm{Pt} / \mathrm{SrTiO}_{3} / \mathrm{Pt}$ structure, a large reduction of electrical resistance is induced for the structure, and oxygen defects are introduced at the interfacial part of $\mathrm{SrTiO}_{3}$. In the resistance decrease by voltage applications, simultaneous occurrence of oxidation and reduction of Ta scavenging layer is shown by EELS analyses from the low-loss spectra. The EELS and scanning transmission electron microscopy observations demonstrate that oxygen scavenging by Ta layer is an interfacial phenomenon where the redox reactions occur at the whole part of the interface. In addition, Pt electrode of the structure, which is chemically inert for oxidation, is revealed to have significant effects in the scavenging processes.

\section{INTRODUCTION}

At a metal/oxide interface, oxygen defects are commonly formed into the oxide through redox reactions between the two layers. The application usage of interfacial oxygen defects into electronic devices have been gaining increasing attention because of their rich chemical and electronic functionalities [1]. Especially, extensive efforts have been devoted toward the control of interfacial oxygen defects in the recent developments of redox-based resistive switching memories or valence change memories [2].

For obtaining their switching operations, oxygen defects have been intentionally introduced into the oxide layer of resistive switching devices, and the introduction has often been made by redox reactions with a reactive electrode material such as $\mathrm{Al}$ and $\mathrm{Ti}[3,4]$. In addition to the conventional method, an improved strategy for defect formation has been recently proposed: a thin interfacial layer of reactive metal between an inert electrode and an oxide, i.e., "scavenging" layer has started to be used in many devices [5-10]. Previous studies supposed that the use of scavenging layer offers high controllability of defect concentrations and easy induction of resistive switching phenomena, and the effectiveness of the layer has been suggested through theoretical calculations [6,8] and evaluations of device characteristics [5,7].

However, the mechanism of oxygen scavenging is still not well understood in most of metal/oxide systems because of the difficulty in observing the oxygen migration in scavenging layers with a nanoscale dimension ( $\leq 10$ nm-thick). For example, a 
controversial issue in the mechanism is whether oxygen scavenging is an intrinsically uniform or local phenomenon. In some works, oxygen scavenging has been considered as a uniform phenomenon, where scavenging layer is uniformly oxidized at the interface both with and without voltage applications [8]. On the other hand, several recent experiments have suggested that oxygen scavenging is a local phenomenon, where scavenging material is preferentially oxidized at a narrow part of the interface by voltage applications [6,10].

To answer such issues and establish an effective method of oxygen scavenging, we performed nanoscopic investigations on defect migrations in a resistive switching oxide of $\mathrm{SrTiO}_{3}$ (STO) with a scavenging layer of Ta. The structure and composition of Ta/STO interfaces was analyzed by high-resolution transmission electron microscopy (HRTEM) and electron energy-loss spectroscopy (EELS), and the impacts of voltage applications were also examined.

\section{EXPERIMENTAL DETAILS}

For the observations of oxygen scavenging phenomenon, we fabricated test structures of $\mathrm{Pt}(100 \mathrm{~nm}) / \mathrm{Ta}(10 \mathrm{~nm}) / \mathrm{STO}(100 \mathrm{~nm}) / \mathrm{Pt}$ [figure 1(a)] by sputtering method. The depositions were performed at room temperature in Ar atmosphere for all layers, and amorphous STO layer was formed in the structures. Pt bottom electrode and STO layer were deposited on $\mathrm{Ti}(10 \mathrm{~nm}) / \mathrm{SiO}_{2} / \mathrm{Si}$ substrates. After the deposition, square-shaped cells with dimensions of $100 \square \mathrm{m} \times 100 \square \mathrm{m}$ were formedon the STO layer by depositing Ta scavenging layer and Pt top electrode through a shadow mask.

HRTEM and EELS measurements were performed for the structures on a Titan 3 G2 60-300 (FEI, Hillsboro, OR, USA). Transport properties of the structures were measured with a B1500 semiconductor parameter analyzer (Keysight Co, Santa Rosa, CA, USA). Voltages were applied from the top Pt electrode and the bottom Pt electrode was grounded in the measurements.

Amorphous STO has been recently reported to be a good resistive switching material having high-performance memory characteristics [5]. In addition, amorphous STO has high structural uniformity with no inclusion of grain boundary and dislocation, which often confines the migration path of oxygen defects. We can thus expect the use of amorphous STO layer offers uniform migrations of oxygen defects in the structures, which allow us to intrinsically observe the processes of oxygen scavenging. The amorphous nature of fabricated STO layers were confirmed both by TEM and X-ray diffraction measurements using a D8 Discover (Bruker AXS Inc., Madison, WI, USA). In addition, Ta has been recently predicted to have a significant oxygen-scavenging function, which makes the formation of conducting channels easier [8]. We thus developed $\mathrm{Pt} / \mathrm{Ta} / \mathrm{STO} / \mathrm{Pt}$ structures as a suitable platform for investigating defect migrations in oxygen scavenging.

\section{RESULTS AND DISCUSSION}

TEM observations for the Pt/Ta/STO/Pt structures showed that the deposited amorphous STO layer has a uniform structure, and no grain boundary or dislocation is included [figure 1(b)]. The TEM results also showed that the structure of Ta scavenging layer is uniform. Therefore, we can expect that detailed observations of oxygen migrations are possible at the Ta/STO interfaces.

We observed that the Ta interfacial layer effectively works as an oxygen scavenging material in the current-time $(I-t)$ characteristics [figure 1(c)]. When no 

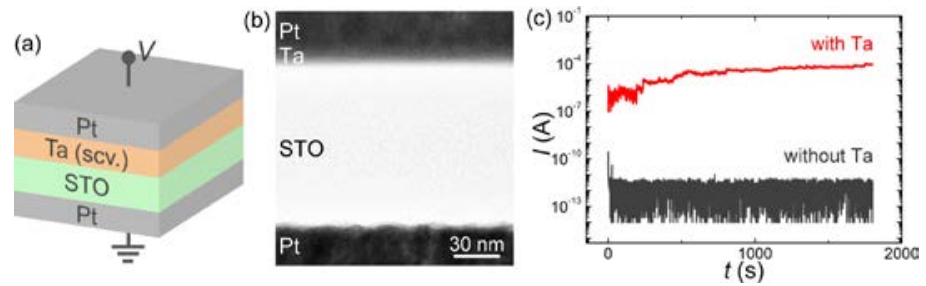

Figure 1. (a) Schematic illustration and (b) TEM image of a $\mathrm{Pt} / \mathrm{Ta} / \mathrm{STO} / \mathrm{Pt}$ structure. (c) $I-t$ characteristics of a $\mathrm{Pt} / \mathrm{Ta} / \mathrm{STO} / \mathrm{Pt}$ and $\mathrm{Pt} / \mathrm{STO} / \mathrm{Pt}$ structure. Constant voltages of $+12.5 \mathrm{~V}$ and $+20.0 \mathrm{~V}$ were applied for the $\mathrm{Pt} / \mathrm{Ta} / \mathrm{STO} / \mathrm{Pt}$ and $\mathrm{Pt} / \mathrm{STO} / \mathrm{Pt}$, respectively.

(a)

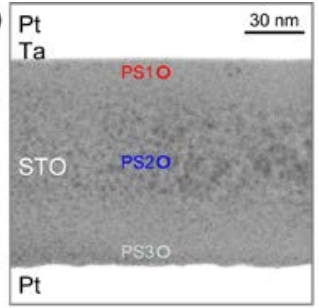

(c)

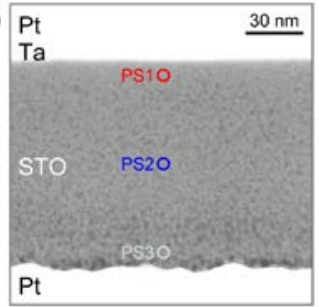

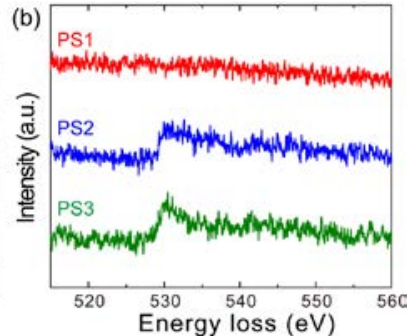

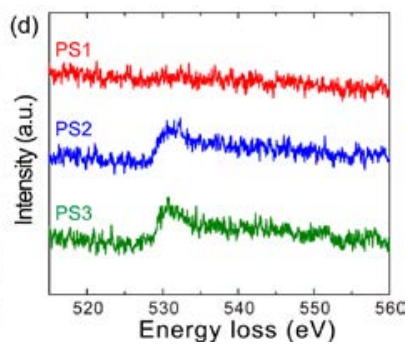

Figure 2. HAADF-STEM images and EEL spectra of the O-K edge for Pt/Ta/STO/Pt structures (a), (b) in the initial state and (c), (d) after resistance decrease to $50 \mathrm{k} \square$ by voltage application of $V=+15 \mathrm{~V}$.

scavenging layer is inserted, the STO-based structures were shown to be highly insulating. Figure 1(c) shows that the resistance of the Pt/STO/Pt structure is $\geqslant 2 \mathrm{~T}$, and no change is induced even by the application of a high constant voltage of $+20 \mathrm{~V}$. This suggests that our STO layer has an insufficient defect concentration for showing resistive switching, and the concentration is not sufficiently increased by voltage applications. On the other hand, large changes in the resistance is induced for the STO structures by inserting Ta scavenging layers. In $\mathrm{Pt} / \mathrm{Ta} / \mathrm{STO} / \mathrm{Pt}$ structures, the initial resistance is largely decreased to $0.4 \mathrm{G} \square$ [figure 1(c)]. This indicates that Ta layer forms a significant amount of oxygen defects into STO through interfacial reactions without applying external voltages. In addition, we observed that the resistance of $\mathrm{Pt} / \mathrm{Ta} / \mathrm{STO} / \mathrm{Pt}$ structure gradually decreases under application of a constant voltage [figure 1(c)]. After the voltage application, persistent decrease in the resistance to $\sim 0.1 \mathrm{M} \square$ was obtained in the structure. These results showed that Ta scavenging layer increased the oxygen defect concentration in STO layer through the electrochemical reactions with it. 
For the $\mathrm{Pt} / \mathrm{Ta} / \mathrm{STO} / \mathrm{Pt}$ structures before and after resistance decrease, we performed EELS analyses of the internal oxygen distributions. Figures 2(b) and 2(d) show the O-K edge EEL spectra for STO layers in the initial state and after resistance decrease. In the initial structure, a single-peak structure was observed for the O-K edge [figure 2(b)] in the middle (PS2) and bottom (PS3) parts of the STO layer [figure 2(a)]. Fine structures for the O-K edges of STO, which are proportional to the unoccupied hybridized density of states [11,12], are nearly lost in the spectra. This behavior suggests that oxygen atoms in the STO layer are highly disordered by the amorphous structure and nonstoichiometry of oxygen. At the top part of the STO layer (PS1), the single peak observed at $530 \mathrm{eV}$ was also diminished in the spectrum [figure 2(b)]. Formation of a higher concentration of oxygen defect by Ta scavenging layer is suggested for the top interface part. After resistance decrease to $50 \mathrm{k} \square$ nearly no change from the initial state was observed both in the high-angle annular dark field scanning transmission electron microscopy (HAADF-STEM) image [figure 2(c)] and O-K edge EEL spectrum [figure 2(d)]. These results suggest that oxygen defects were not in the bulk of STO layer and more likely to be introduced into the top interfacial part around PS1 after the resistance decrease.

To investigate the details of interfacial defect formation in oxygen scavenging, we performed EELS analyses for Ta layers. Although tantalum oxides have been less investigated by EELS, a recent study has shown that the low-loss spectra are sensitively affected by the oxidation states, and can be a good indicator of the oxidation extent $[13,14]$. Low-energy EEL spectra for Pt/Ta/STO interfaces before and after resistance decrease are shown in figures 3(b) and 3(d). For the initial interface, a peak was observed at $22 \mathrm{eV}$ for the upper and middle parts of the layer (PT2 and PT3), but a multiple peak structure was observed for the interfacial parts near Pt electrode (PT1). Based on literature [13,14], we can consider that the peak at $22 \mathrm{eV}$ (marked as "b") and the satellite structures ("a", "c" and "d") originate in metallic Ta and the oxides $\left(\mathrm{TaO}, \mathrm{TaO}_{2}\right.$, and $\mathrm{Ta}_{2} \mathrm{O}_{5}$ ), respectively. Therefore, although the entire part of the Ta layer is assumed to be oxidized by scavenging because peaks "a" and "c" are also included in the spectrum for the internal parts (PT2 and PT3), the EELS spectra showed that a larger extent of
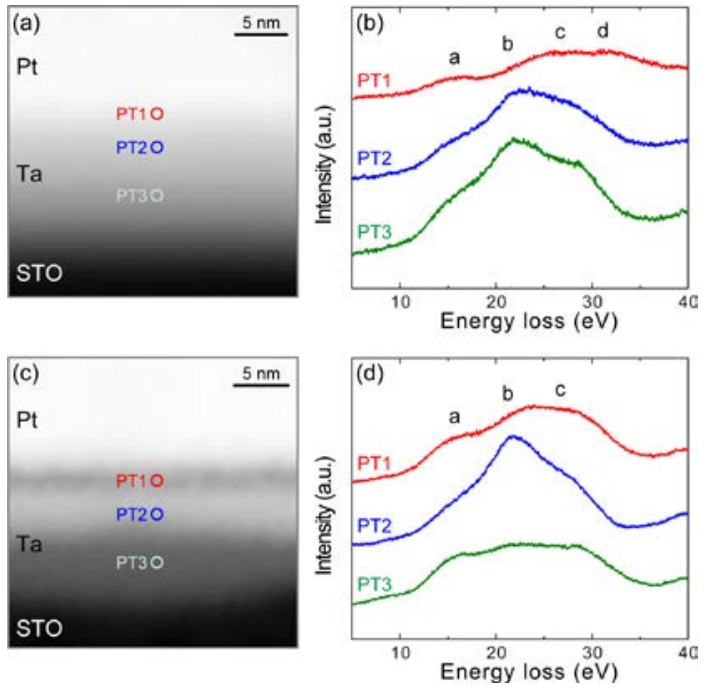
(a)

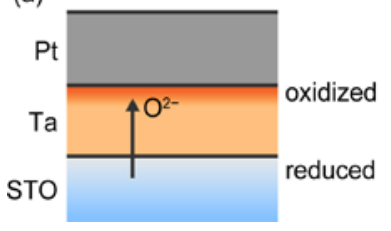

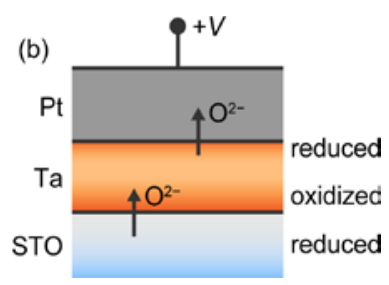

Figure 4. Schematic illustrations of oxygen migration in the scavenging phenomenon by Ta interfacial layer for STO (a) before and (b) after voltage application.

oxidation occurs at the top interface away from the STO layer in the initial state.

After induction of resistance decrease, clear changes were observed in the HAADF-STEM image of Ta layer [figure 3(c)]. In the images, dark contrast appeared both in the lower part and at the top interface with Pt. We observed that the peak structure in the EEL spectrum became broader for the PT3-part in the bottom darkened region of Ta layer [figure 3(d)]. The occurrence of oxygen migration from the STO layer was suggested for this part, and no large change in the oxidation state was observed in the upper part of PT2. At the interfacial part of PT1 darkened in the HAADF-STEM image, interestingly, the broad peak structure in EELS profile became sharpened after the resistance decrease, and a larger peak was constructed at "b" of $22 \mathrm{eV}$. This result shows that reduction was induced for the top interface of Ta layer by the resistance decrease rather than oxidation.

Our results showed that oxygen scavenging by Ta layer is an interfacial phenomenon both in the pristine state and under voltages. In the initial structure before voltage application, Ta scavenging layer is uniformly oxidized due to the scavenging function, and the interfacial part of STO layer is reduced as shown in figure 4(a). The very interface of Ta layer near Pt/Ta will be specifically oxidized by Pt electrode due to its catalytic function [15]. When voltages are applied to Pt, oxygen ions drift toward top $\mathrm{Pt}$, and STO is electrochemically reduced [figure 4(b)]. As observed as contrast changes in the HAADF-STEM image [figure 3(c)], the electrochemical oxidation of Ta scavenging layer is considered to occur at nearly all the interfacial area. The upper part of Ta layer, on the other hand, was shown to be reduced during the voltage application. This indicates that oxygen evolution into Pt electrode $[15,16]$ is also induced in the electrochemical scavenging processes. As the results, oxygen concentrations in Ta scavenging layer forms complex gradients in the thickness direction both in the initial sate and after voltage applications, and the oxidation process is also strongly affected by the electrode material $(\mathrm{Pt})$.

\section{CONCLUSIONS}

Our EELS analyses revealed that interfacial (non-local) oxygen scavenging is possible at Ta/STO interfaces both with and without voltage applications. The use of Ta scavenging layer was shown to form interfacial defects into STO layers and induces significant decrease in the resistance by voltage applications. For Ta scavenging layers, we found that both oxidation and reduction is induced by voltage applications in a parallel way. Complex concentration profiles of oxygen were thus formed in the Ta/STO structures after scavenging. Moreover, our results showed that Pt electrodes also has significant impacts on the scavenging mechanism through the catalytic and evolution effects. These findings will provide important insights into future developments of 
redox-based resistive switching memory and other related oxide devices, where the control of oxygen defect concentrations has a critical importance in the operation.

\section{ACKNOWLEDGMENTS}

This work was financially supported in part under KAKENHI by the Japan Society for the Promotion of Science (JSPS) (Nos. 16K18073, 16H04339, 15H01706). The Nippon Sheet Glass Foundation for Materials Science and Engineering and Scholar Project of Toyota Physical and Chemical Research Institute are also acknowledged for their financial support of this work. Part of the experiments was performed under the Nanotechnology Platform Program (Hokkaido Univ.) organized by the Ministry of Education, Culture, Sports, Science and Technology (MEXT), Japan.

\section{REFERENCES}

1. A. Picone, M. Riva, A. Brambilla, A. Calloni, G. Bussetti, M. Finazzi, F. Ciccacci and L. Duò, Surf. Sci. Rep. 71, 32-76 (2016).

2. A. Sawa, Mater. Today 11, 28-36 (2008).

3. C. B. Lee, B. S. Kang, A. Benayad, M. J. Lee, S.-E. Ahn, K. H. Kim, G. Stefanovich, Y. Park and I. K. Yoo, Appl. Phys. Lett. 93, 042115 (2008).

4. K.-L. Lin, T.-H. Hou, J. Shieh, J.-H. Lin, C.-T. Chou and Y.-J. Lee, J. Appl. Phys. 109, 084104 (2011).

5. H. Nili, S. Walia, S. Balendhran, D. B. Strukov, M. Bhaskaran and S. Sriram, Adv. Funct. Mater. 24, 6741-6750 (2014).

6. Y. Guo and J. Robertson, Appl. Phys. Lett. 105, 223516 (2014).

7. M. Prezioso, F. Merrikh-Bayat, B. D. Hoskins, G. C. Adam, K. K. Likharev and D. B. Strukov, Nature 521, 61-64 (2015).

8. X. Zhong, I. Rungger, P. Zapol, H. Nakamura, Y. Asai and O. Heinonen, Phys. Chem. Chem. Phys. 18, 7502-7510 (2016).

9. W. Kim, S. Menzel, D. J. Wouters, Y. Guo, J. Robertson, B. Roesgen, R. Waser and V. Rana, Nanoscale 8, 17774-17781 (2016).

10. U. Celano, J. Op de Beeck, S. Clima, M. Luebben, P. M. Koenraad, L. Goux, I. Valov and W. Vandervorst, ACS. Appl. Mater. Interfaces 9, 10820-10824 (2017).

11. D. A. Muller, N. Nakagawa, A. Ohtomo, J. L. Grazul and H. Y. Hwang, Nature 430, 657-661 (2004).

12. H. Du, C.-L. Jia, A. Koehl, J. Barthel, R. Dittmann, R. Waser and J. Mayer, Chem. Mater. 29, 3164-3173 (2017).

13. J. K. N. Sharma, B. R. Chakraborty and S. Bera, Surf. Sci. 285, 237-242 (1993).

14. G.-S. Park, Y. B. Kim, S. Y. Park, X. S. Li, S. Heo, M.-J. Lee, M. Chang, J. H. Kwon, M. Kim, U.-I. Chung, R. Dittmann, R. Waser and K. Kim, Nat. Commun. 4, 2382 (2013).

15. D. S. Jeong, H. Schroeder, U. Breuer and R. Waser, J. Appl. Phys. 104, 123716 (2008).

16. D. Cooper, C. Baeumer, N. Bernier, A. Marchewka, C. La Torre, R. E. Dunin-Borkowski, S. Menzel, R. Waser and R. Dittmann, Adv. Mater. 29, 1700212 (2017). 